\title{
A modified ultra-long pituitary downregulation protocol improved endometrial receptivity and clinical outcome for infertile patients with polycystic ovarian syndrome
}

\author{
FEI GONG ${ }^{1-3}, \mathrm{XIHONG} \mathrm{LI}^{2}$, SHUNJI ZHANG ${ }^{2}$, HAINAN MA ${ }^{2}$, \\ SUFEN CAI ${ }^{2}, \mathrm{JUAN}^{2}{ }^{2}, \mathrm{GE} \mathrm{LIN}{ }^{1-3}$ and GUANGXIU LU ${ }^{1-3}$ \\ ${ }^{1}$ Institute of Reproductive and Stem Cell Engineering, Central South University, Changsha, Hunan 410083; \\ ${ }^{2}$ Department of Reproductive Medicine, Reproductive and Genetic Hospital of Citic-Xiangya, Changsha, Hunan 410078; \\ ${ }^{3}$ Key Laboratory of Human Stem Cell \& Reproductive Engineering, Ministry of Health, Changsha, Hunan 410008, P.R. China
}

Received September 18, 2014; Accepted July 31, 2015

DOI: $10.3892 /$ etm.2015.2769

\begin{abstract}
There are currently various protocols for in vitro fertilization (IVF). For patients with polycystic ovarian syndrome (PCOS), an optimized protocol for the downregulation of pituitary follicle stimulating hormone and luteinizing hormone via gonadotropin-releasing hormone agonist (GnRHa) remains a challenge. In the present study, the primary endpoint of an ultra-long and a conventional long GnRHa protocol for intracytoplasmic sperm injection/IVF treatments of patients with PCOS was retrospectively compared. In the modified ultra-long protocol group, endometrial thickness, morphology, and blood flow were significantly improved, as compared with in the conventional long protocol group. Furthermore, the serum progestogen $(\mathrm{P})$ concentrations and P/estrogen (E2) [(Px1,000/E2)] ratio on the day of human chorionic gonadotrophin administration were significantly decreased in the modified ultra-long downregulation group, whereas the pregnancy and implantation rates were significantly higher. There were no significant differences in the average number of obtained oocytes, good quality embryo rates, cancel rates, fertilization rates, abortion rates, serious ovarian hyperstimulation syndrome incidences, ectopic pregnancy rates or gonadotropin ( $\mathrm{Gn}$ ) dosages between the two groups. These results suggest that the modified ultra-long protocol plus human menopausal Gn medication may be superior to the conventional long protocol, and may lead to improved implantation and pregnancy outcomes for infertile patients with PCOS.
\end{abstract}

Correspondence to: Professor Guangxiu Lu, Institute of Reproductive and Stem Cell Engineering, Central South University. 932 Lushan South Road, Changsha, Hunan 410083, P.R. China E-mail: luguangxiubm@163.com

Key words: polycystic ovarian syndrome, ultra-long downregulation protocol, endometrial receptivity

\section{Introduction}

Polycystic ovarian syndrome (PCOS) is a common endocrine disorder in women of childbearing age. Characteristic clinical presentations include: Menstrual disorders, oligoovulation or anovulation, infertility, hirsutism, obesity, ovarian enlargement, and irregular serum hormone concentrations (1). Patients with PCOS account for 5-10\% of women of childbearing age, $70-80 \%$ of patients with ovulation disorder, and $50 \%$ of patients who are undergoing assisted reproductive technologies (2).

Due to the complex endocrine conditions associated with PCOS, the induction of controlled ovulation for in vitro fertilization (IVF) or intracytoplasmic sperm injection (ICSI) is challenging. This is largely due to the very narrow response range to gonadotropin $(\mathrm{Gn})$, ovarian hyperstimulation syndrome (OHSS), and a surge in endogenous luteinizing hormone (LH), which may affect the quality of oocytes and reduce clinical pregnancy rates (3-5). To date, no exact optimization scheme exists for patients with PCOS (6). Currently, the majority of reproductive centers use traditional long protocols to inhibit an endogenous premature LH surge and prevent premature luteinized follicles, in order to induce high quality oocytes and successful pregnancies $(7,8)$. As such, the pregnancy rates of patients with PCOS who receive the long pituitary downregulation protocol are not always ideal; some patients exhibit poor outcomes due to insufficient downregulation, and premature endogenous LH surge, even with a long duration of high dose $\mathrm{Gn}$. To date, the ultra-long protocol has predominantly been used for infertile patients with endometriosis, with noteworthy outcomes (9). In the present study, a modified ultra-long protocol for patients with PCOS was implemented. Data were retrospectively summarized between September 2011 and April 2012 in our assisted reproductive technology center in the Reproductive \& Genetic Hospital of Citic-Xiangya (Changchun, China). Patients with PCOS were divided into modified ultra-long protocol or conventional long protocol groups, and endogenous LH levels, endometrial receptivity, and the clinical outcomes of the two groups were compared. 


\section{Materials and methods}

Patients. A total of 147 patients admitted to the Reproductive \& Genetic Hospital of Citic-Xiangya were recruited between September 2011 and April 2012 undergoing IVF/ICSI, were divided into two groups: An ultra-long protocol group $(n=75)$ and a long protocol group $(\mathrm{n}=72)$. The exclusion criteria were: Related disorders, $>38$ years old, endometriosis, uterine malformation, untreated hydrosalpinx, intrauterine adhesions, and a history of endometriosis. PCOS was diagnosed according to the Rotterdam criteria (10) and patients were selected based on at least two of the following three features: i) Oligoovulation or anovulation, ii) clinical and/or biochemical signs of hyperandrogenism, or iii) polycystic ovaries with congenital adrenal hyperplasia, excluding Cushing's syndrome and tumor-related testosterone concentration elevations. The research protocol was approved by the Ethics Committee of the Central South University (Changsha, China) and the Reproductive \& Genetic Hospital of Citic-Xiangya. All participants provided their written informed consent to participate in the present study.

Treatments. The starting doses of Gn releasing hormone agonist (GnRHa) were chosen according to the patient's age, history and body weight. All patients received Dane-35 (Schering GmbH und Co. Produktions KG, Bayer, Germany) for one complete menstrual cycle from day 3 .

Routine long pituitary downregulation protocol. On day 20 of the patient's menstrual cycle $1.5-1.875 \mathrm{mg}$ GnRHa was intramuscularly injected. After a period of 13-20 days, following confirmation of pituitary-ovarian suppression , 75U-150 IU/d recombinant follicle stimulating hormone ( $\mathrm{rFSH}$ ) (Gonal-F or Puregon; Merck Serono S.A., Coinsins, Switzerland) was administered for 4-5 days. Human chorionic Gn (hCG) was injected following confirmation of adequate follicle stimulation by ultrasound and hormone concentrations.

Modified ultra-long pituitary downregulation protocol. On day 20 of the patient's first menstrual cycle $1.5-1.875 \mathrm{mg} \mathrm{GnRHa}$ was intramuscularly injected. This was repeated on day 21 of the following menstrual cycle. After 13-20 days, following confirmation of pituitary-ovarian suppression, human menopausal gonadotropin (hMG, 75 U-375 U/d, Menopur; Ferring Pharmaceuticals, Kiel, Germany) was injected for 4-5 days. hCG was injected following confirmation of adequate follicle stimulation by ultrasound and hormone concentrations.

Evaluation criteria for follicle stimulation. Follicle development status was determined by ultrasonography, combined with serum hormone concentration determinations. Controlled ovarian hyperstimulation $(\mathrm{COH})$ was adapted using a $\mathrm{Gn}$ step-up, step-down or withdrawal scheme according to estrogen (E2), progestogen (P), and luteinizing hormone (LH) levels, and vaginal ultrasound results. The criteria for adequate pituitary-ovarian suppression were: $\mathrm{E} 2<40 \mathrm{pg} / \mathrm{ml}, \mathrm{P}<0.8 \mathrm{ng} / \mathrm{ml}$ and $\mathrm{LH}<3 \mathrm{mIU} / \mathrm{ml}$ serum concentrations; as well as endometrium thickness $<5 \mathrm{~mm}$ and, no follicle or corpus luteum cyst $\geq 10 \mathrm{~mm}$ present in the ovary. $\mathrm{rFSH}$ or hMG was administered to achieve egg stimulation until follicles $\geq 18 \mathrm{~mm}$ accounted for $60-70 \%$ of follicles $>14 \mathrm{~mm}$, or follicles $\geq 20 \mathrm{~mm}$ accounted for $40-50 \%$ of follicles $>14 \mathrm{~mm}$. Patients were scheduled for oocyte retrieval $35-36 \mathrm{~h}$ following hCG injection. E2 per every $14 \mathrm{~mm}$ follicle was $200-300 \mathrm{pg} / \mathrm{ml}$. Indications and techniques for oocyte aspiration, oocyte and embryo culture, insemination, ICSI, assisted hatching and embryo transfer (ET) were based on the routine of the center (ISO 9001 Certification) (11).

Determinations of endometrial change. The endometrial thickness, morphology alterations, and blood flow of the two groups were assessed by vaginal color ultrasound on the day of hCG injection and the day of ET. GE V730-expert color Doppler ultrasonic diagnostic apparatus (GE Healthcare Life Sciences, Little Chalfont, UK) was used with an intra-cavity probe frequency of 5-9 MHz. The operator and settings remained constant throughout the study, and test indices were longitudinal endometrial thickness and endometrial morphology, which were assessed using the classification system outlined by Gonen and Casper (12), as follows; Type A, three linear or multilayer endometrium, echogenic outer and central areas and hypoechoic or dark inner areas, and significant uterine cavity midline echo; Type $\mathrm{B}$, the middle area exhibits an isolated echo-like uterine myometrium image, and the uterine cavity midline echo is not clear; Type C, the endometrium is homogeneously echogenic, without uterine cavity midline echo. Endometrial blood flow was categorized according to Chien et al (13); Type A, blood flow could be monitored as endometrial and subendometrial; Type B, blood flow could only be monitored as subendocardial blood flow; and Type C, Endometrium and endometrial blood flow could not be detected.

Outcome parameters. Serum hormone concentrations of the both groups were evaluated prior to GnRHa stimulation and on the day of hCG administration. The mean number of retrieved oocytes, fertilization rate, good quality embryo rate, cycle cancellation rate, the number of transferred embryos, implantation rate, pregnancy rate, miscarriage rate following transplantation, severe OHSS incidence, the incidence of ectopic pregnancy, the GnRHa dosage, and the days of drug administration were compared between the two groups.

Statistical analyses. Sigmastat software, version 3.5 (Systat Software Inc., San Jose, CA, USA) was used to carry out statistical analyses. Values are expressed as mean \pm standard deviation, or number and percentage. Comparison of quantitative variables between groups was achieved by one-way analysis of variance. Homogeneity of variance and normality of data were estimated using Levene and Kolmogorov-Smirnov tests, respectively. Qualitative variables were analyzed by $\chi^{2}$ test. $\mathrm{P}<0.05$ was considered to indicate a statistically significant difference.

\section{Results}

Age, infertility duration (years), body mass index (BMI), and basal FSH and LH values were compared between the two groups and no significant differences were detected (Table I). Table II presents the comparisons between clinical outcomes of the two protocols. Within the ultra-long protocol group, the LH level on the day prior to $\mathrm{Gn}$ administration, and the P level 
Table I. Basic clinical features of the patients with polycystic ovarian syndrome.

\begin{tabular}{lrrr}
\hline Characteristics & Ultra-long protocol $(\mathrm{n}=75)$ & Long protocol $(\mathrm{n}=72)$ & $\mathrm{P}-\mathrm{value}$ \\
\hline Age (years) & $28.01 \pm 2.03$ & $27.89 \pm 2.91$ & 0.7652 \\
Infertility duration (years) & $4.49 \pm 2.43$ & $3.88 \pm 2.44$ & 0.1262 \\
BMI $\left(\mathrm{kg} / \mathrm{m}^{2}\right)$ & $22.61 \pm 2.85$ & $21.71 \pm 2.85$ & 0.0580 \\
Basal FSH $(\mathrm{mIU} / \mathrm{ml})$ & $5.89 \pm 1.21$ & $6.12 \pm 1.17$ & 0.2460 \\
Basal LH (mIU/ml) & $8.41 \pm 5.08$ & $7.58 \pm 6.20$ & 0.3753
\end{tabular}

BMI, Body mass index; FSH, follicle stimulating hormone; LH, luteinizing hormone.

Table II. Comparison of clinical outcomes of patients with polycystic ovarian syndrome treated with two protocols.

\begin{tabular}{|c|c|c|c|}
\hline Variables & Ultra-long protocol $(\mathrm{n}=75)$ & Long protocol $(n=72)$ & P-value \\
\hline $\mathrm{LH}$ level on the day prior to $\mathrm{Gn}(\mathrm{mIU} / \mathrm{ml})$ & $0.63 \pm 0.69$ & $1.89 \pm 0.87$ & $<0.0001^{\mathrm{a}}$ \\
\hline Dosage of Gn (IU) & $2104.9 \pm 908.92$ & $2020.76 \pm 899.76$ & 0.5737 \\
\hline Duration of Gn (days) & $11.52 \pm 2.15$ & $11.85 \pm 2.13$ & 0.3562 \\
\hline P level on hCG day (ng/ml) & $0.63 \pm 0.22$ & $0.77 \pm 0.23$ & $0.0002^{\mathrm{a}}$ \\
\hline E2 level on hCG day (pg/ml) & $3707.29 \pm 1307.97$ & $3667.29 \pm 1621.14$ & 0.8698 \\
\hline Px 1,000/E2 on HCG day & $0.19 \pm 0.08$ & $0.25 \pm 0.13$ & $0.0006^{\mathrm{a}}$ \\
\hline LH level on HCG day (mIUml) & $1.40 \pm 0.45$ & $1.29 \pm 0.51$ & 0.1558 \\
\hline Mean number of oocytes retrieved & $14.63 \pm 5.96$ & $15.58 \pm 5.00$ & 0.2928 \\
\hline Fertilization rate $(\%)$ & $84.11 \pm 17.16$ & $82.26 \pm 14.95$ & 0.4865 \\
\hline Good quality embryo rate (\%) & $71.13 \pm 25.10$ & $66.52 \pm 25.15$ & 0.2679 \\
\hline Implantation rate $(\%)$ & $59.56(81 / 136)$ & $44.09(56 / 127)$ & $0.012^{\mathrm{a}}$ \\
\hline Cancel rate $(\%)$ & $9.33(7 / 75)$ & $13.89(10 / 72)$ & 0.388 \\
\hline Number of transferred embryos & $2.0 \pm 0$ & $2.05 \pm 0.28$ & 0.1595 \\
\hline Clinical pregnancy rate per transfer $(\%)$ & $77.94(53 / 68)$ & $61.29(38 / 62)$ & $0.039^{\mathrm{a}}$ \\
\hline Severe OHSS rate $(\%)$ & $1.33(1 / 75)$ & $2.78(2 / 72)$ & 0.536 \\
\hline Abortion rate $(\%)$ & $4.76(3 / 53)$ & $4.76(2 / 38)$ & 1.000 \\
\hline Ectopic pregnancy rate $(\%)$ & $0(0 / 53)$ & $2.38(1 / 38)$ & 0.218 \\
\hline
\end{tabular}

LH, lutenizing hormone; Gn, gonadotropin; P, progestogen; E2, estrogen; hCG, human chorionic gonadotophin; OHSS, ovarian hyperstimulation syndrome. ${ }^{\mathrm{a}} \mathrm{P}<0.05$.
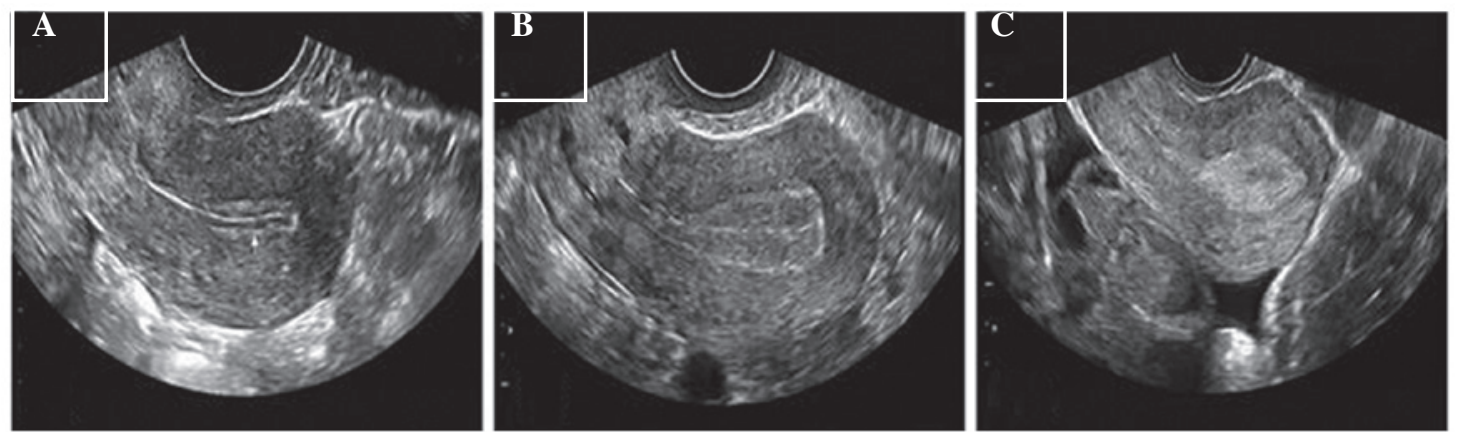

Figure 1. Classification of endometrial morphology. (A) Type A: Three linear or multilayer endometrium; the outer and central areas were echogenic, and the inner area was hypoechoic or dark, the uterine cavity midline echo was obvious. (B) Type B: The middle area produced isolated echos such as in a uterine myometrium image, and the uterine cavity midline echo was not clear. (C) Type C: The endometrium was homogeneously echogenic, without a uterine cavity midline echo.

$(\mathrm{ng} / \mathrm{ml})$ and $\mathrm{P} / \mathrm{E} 2(\mathrm{P}(\mathrm{ng} / \mathrm{ml}) \times 1,000 / \mathrm{E} 2(\mathrm{pg} / \mathrm{ml}))$ ratio on the day of hCG administration were significantly lower, as compared with that of the long protocol group $(\mathrm{P}<0.05)$. Furthermore, the implantation and clinical pregnancy rates of the ultra-long 
Table III. Endometrial thickness, morphology, and blood flow following treatment with the two protocols.

\begin{tabular}{|c|c|c|c|}
\hline Endometrial characteristics & $\begin{array}{l}\text { Ultra-long protocol } \\
\qquad(\mathrm{n}=75)\end{array}$ & $\begin{array}{l}\text { Long protocol } \\
\qquad(\mathrm{n}=72)\end{array}$ & P-value \\
\hline \multicolumn{4}{|l|}{ Day of hCG administration } \\
\hline Endometrial thickness (mm) & $2.67 \pm 1.67$ & $11.78 \pm 2.30$ & 0.0092 \\
\hline \multicolumn{4}{|l|}{ Endometrial morphology (\%) } \\
\hline A & $32.0(24 / 75)$ & $23.6(17 / 72)$ & 0.257 \\
\hline $\mathrm{B}$ & $57.3(43 / 75)$ & $43.1(31 / 72)$ & 0.083 \\
\hline $\mathrm{C}$ & $10.7(8 / 75)$ & $33.3(24 / 72)$ & 0.001 \\
\hline \multicolumn{4}{|l|}{ Endometrial blood flow (\%) } \\
\hline A & $50.7(38 / 75)$ & $36.1(26 / 72)$ & 0.075 \\
\hline $\mathrm{B}$ & $49.3(37 / 75)$ & $56.9(41 / 72)$ & 0.278 \\
\hline $\mathrm{C}$ & $0(0 / 75)$ & $6.9(5 / 72)$ & 0.020 \\
\hline \multicolumn{4}{|l|}{ Day of ET } \\
\hline Endometrial thickness (mm) & $12.91 \pm 2.01$ & $11.97 \pm 2.85$ & 0.0226 \\
\hline \multicolumn{4}{|l|}{ Endometrial morphology $(\%)$} \\
\hline A & $0(0 / 75)$ & $0(0 / 72)$ & 1.000 \\
\hline $\mathrm{B}$ & $0(0 / 75)$ & $0(0 / 72)$ & 1.000 \\
\hline $\mathrm{C}$ & $100(75 / 75)$ & $100(72 / 72)$ & 1.000 \\
\hline \multicolumn{4}{|l|}{ Endometrial blood flow (\%) } \\
\hline A & $32.0(24 / 75)$ & $23.6(17 / 72)$ & 0.257 \\
\hline $\mathrm{B}$ & $49.3(37 / 75)$ & $37.5(27 / 72)$ & 0.148 \\
\hline $\mathrm{C}$ & $18.7(14 / 75)$ & $38.9(28 / 72)$ & 0.007 \\
\hline
\end{tabular}

hCG, human chorionic gonadotropin; ET, embryo transfer.
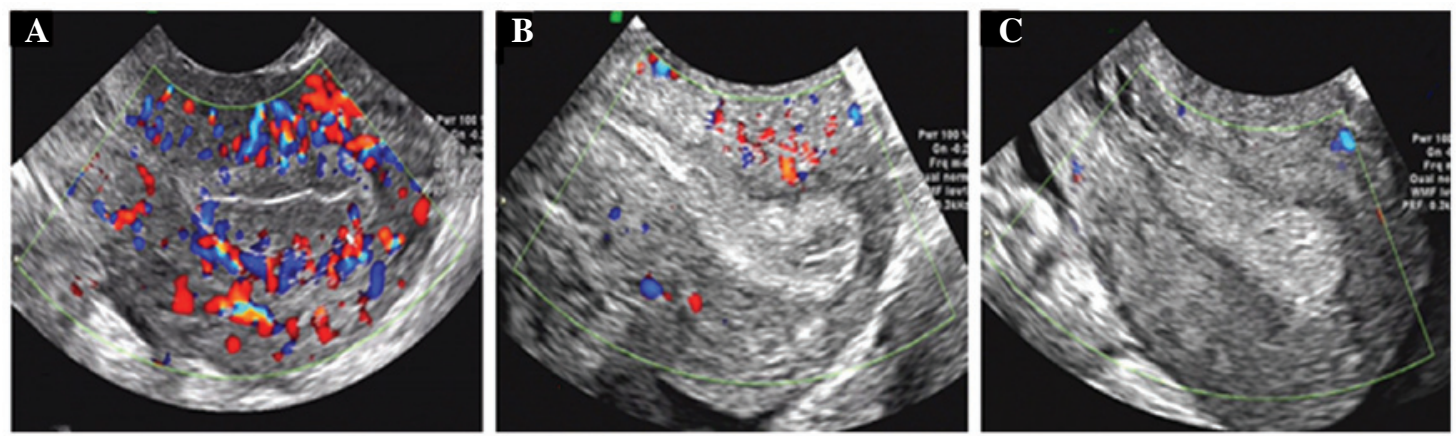

Figure 2. Classification of endometrial and subendometrial blood flows. (A) Type A: Could be monitored to endometrial and subendometrial blood flow; (B) Type B: Could only be monitored to subendocardial blood flow; (C) Type C: Endometrium and endometrial blood flows were not detected.

protocol group were significantly higher, as compared with those of the long protocol group, $(\mathrm{P}<0.05)$. Other parameters included: Gn dosage, duration of $\mathrm{Gn}$, levels of $\mathrm{E} 2$ and $\mathrm{LH}$ on the day of hCG, the mean number of oocytes retrieved, fertilization rates, good quality embryo rates, cycle cancellation rates, the number of transferred embryos, implantation rates, pregnancy rates, miscarriage rates of transplantations, severe OHSS incidences and the incidence of ectopic pregnancies, all of which exhibited no significant differences. The increase in clinical pregnancy rate observed in the ultra-long protocol group may be caused by the optimal $\mathrm{P}$ level and $\mathrm{P} / \mathrm{E} 2$ ratio on the day of hCG administration, which resulted in improved endometrial receptivity. Endometrial morphology and blood flow are shown in Figs. 1 and 2, whereas Table III details the differences in endometrial thickness, morphology, and blood flow between the two protocols. The proportion of patients with type $\mathrm{A}$ and $\mathrm{B}$ endometrial morphology on the day of hCG administration in the ultra-long protocol group was 32.0 and $57.3 \%$, respectively; thus greater than that observed in the long protocol group (23.6 and $43.1 \%$, respectively); however significance was not reached. The proportion of patients with type $\mathrm{C}$ endometrial morphology on the day of hCG application in the ultra-long protocol group (10.7\%) was significantly lower as compared with that of the long protocol 
group $(33.3 \%)(\mathrm{P}=0.001)$. The endometrial morphology of the patients receiving both protocols was type $\mathrm{C}$ on the day of ET. The proportion of patients with types $\mathrm{A}$ and $\mathrm{B}$ endometrial blood flow and sub-endometrial tissue, on the days of hCG medication and of ET was higher in the ultra-long protocol group compared with the long protocol group; however, the difference was not statistically significant. The proportion of patients with type $\mathrm{C}$ endometrial morphology on the days of hCG administration and ET were significantly lower in the ultra-long protocol group compared with the long protocol group.

\section{Discussion}

The present study demonstrated that patients with PCOS treated with an ultra-long protocol pituitary downregulation protocol benefited from improved clinical outcomes, as compared with patients in the long protocol group; this may be associated with a reduction in $\mathrm{P}$ level on the day of $\mathrm{hCG}$ administration. Bosch et al (14) demonstrated that serum $\mathrm{P}$ levels and the ratio of P/E2 on the day of hCG administration may be indicators of an implantation window for optimal endometrial receptivity, which is shifted forward in IVF patients, as compared with women in natural menstruation cycles. Elgindy (15) reported that clinical blastocyst pregnancy rates per transfer were reduced if, on the day of hCG administration, the $\mathrm{P}$ serum levels and the ratio of P/E2 were higher than $1.5 \mathrm{ng} / \mathrm{ml}$ and 0.55 , respectively. Previous studies (16-18) have noted high $\mathrm{P}$ levels on the day of hCG administration and resultant lower clinical and complete pregnancy rates. The results of the present study demonstrated that serum $\mathrm{P}$ levels and the ratio of $\mathrm{P} / \mathrm{E} 2$ on the day of $\mathrm{hCG}$ administration were significantly lower in the ultra-long protocol group, as compared with those in the long protocol group; thus inhibiting the forward shift of the uterine endometrium implantation window and improving endometrial receptivity. LH levels prior to the day of $\mathrm{Gn}$ stimulation reflect downregulation of pituitary suppression. Prior to Gn stimulation, lower LH levels were observed in the ultra-long protocol group compared with the long protocol group. A prior study suggested that the optimum LH threshold range of $0.5-1.5 \mathrm{IU} / 1$ (19). hMG was used to initiate ovulation in the ultra-long protocol, which was a supplement for low LH levels and obtained good pregnancy outcomes.

Previous studies have demonstrated that embryos may be easier to implant, and exhibit improved development on type $\mathrm{A}$ and $\mathrm{B}$ endometrial morphologies, as compared with that of type $\mathrm{C}(6,20,21)$. Järvelä et al $(22)$ suggested that $\mathrm{COH}$ multilayer (three linear) endometrial morphology is more suitable for embryo implantation, with homogeneous endometrial morphology associated with poor IVF outcomes, as non-three linear endometrial morphology can indicate that premature transformation of the endometrium into the secretory phase. In the present study, the percentage of type $\mathrm{C}$ endometrial morphology on the day of hCG medication in the ultra-long protocol group was significantly lower, as compared with that observed in the long protocol group $(\mathrm{P}=0.001)$. The majority of previous studies concur that appropriate thickness of the endometrium is essential for embryo implantation. It has been observed that clinical pregnancy rates increase once the endometrial thickness increases to $>14 \mathrm{~mm}$ on the day of hCG application, whereas implantation and pregnancy rates were almost zero when endometrial thickness was $<6-7 \mathrm{~mm}(21,23-26)$. In the present study, the endometrium of the ultra-long protocol group was thicker on the day of hCG administration, as compared with that of the long protocol group, and the endometrial morphologies on day of ET were type $\mathrm{C}$ in both protocols. These findings demonstrate that the ultra-long protocol led to adequate pituitary downregulation and may have inhibited premature endometrium transformation into the secretory phase, thus inhibiting the forward shift of the endometrial implantation window.

In the present study, the proportion of patients with type A endometrial blood flow and sub endometrial tissue on the days of hCG medication and of ET was higher in the ultra-long protocol group compared with the long protocol group. The proportion of patients with type B endometrial blood flow and sub endometrial tissue on the day of ET was higher in the ultra-long protocol group compared with the long protocol group; however, the difference was statistically insignificant. In previous studies, good sub-endometrial and endometrial perfusion has been reported to be important for successful embryo implantation $(8,13,27)$. Furthermore, previous studies have noted that endometrial blood flow following oocyte retrieval in IVF/ICSI-ET pregnant patients, and in spontaneous pregnancies post-ovulation, was higher than in non-pregnant women $(22,28)$.

Obesity and a high BMI negatively affect embryo quality and endometrial receptivity in assisted reproduction. Pregnancy rates have been shown to be significantly lower in patients with $\mathrm{BMI} \geq 27 \mathrm{~kg} / \mathrm{m}^{2}$, as compared with in patients with $\mathrm{BMI} \leq 25 \mathrm{~kg} / \mathrm{m}^{2}$ and $25-27 \mathrm{~kg} / \mathrm{m}^{2}$ (27,29-31). The ideal BMI of Chinese women is $18-23 \mathrm{~kg} / \mathrm{m}^{2}$ and in the present study, the BMI of patients in the modified ultra-long protocol group was slightly higher, as compared with that in the long protocol group. However, the implantation and pregnancy rates were significantly higher in patients in the ultra-long protocol group, as compared with the long protocol group; therefore, we propose that patients with PCOS with BMIs $>23 \mathrm{~kg} / \mathrm{m}^{2}$ or obesity may be more suitable for the ultra-long protocol.

In conclusion, two injections of small GnRHa doses prior to $\mathrm{COH}$, combined with hMG, significantly increased pregnancy rates. Lower serum $\mathrm{P}$ levels on the day of hCG administration in a modified ultra-long pituitary downregulation protocol may improve pregnancy rates and ameliorate the receptivity of the endometrium, as assessed by endometrial morphology, thickness, and blood flow. It may be hypothesized that a modified ultra-long protocol, coupled with hMG ovarian stimulation, is a preferable treatment for patients with PCOS, especially those with elevated BMIs. Further studies should focus on biological endometrial changes and associated mechanisms in an ultra-long protocol.

\section{References}

1. Sheehan MT: Polycystic ovarian syndrome: Diagnosis and management. Clin Med Res 2: 13-27, 2004.

2. Hart R: Polycystic ovarian syndrome - prognosis and treatment outcomes. Curr Opin Obstet Gynecol 19: 529-535, 2007.

3. Chen ZJ and Shi Y: Polycystic ovary syndrome. Front Med China 4: 280-284, 2010

4. Elnashar AM: Progesterone rise on the day of HCG administration (premature luteinization) in IVF: An overdue update. J Assist Reprod Genet 27: 149-155, 2010. 
5. Pundir J, Sunkara SK, El-Toukhy T and Khalaf Y: Meta-analysis of GnRH antagonist protocols: Do they reduce the risk of OHSS in PCOS? Reprod Biomed Online 24: 6-22, 2012.

6. Orvieto R, Meltcer S, Liberty G, Rabinson J, Anteby EY and Nahum R: Does day-3 LH/FSH ratio influence in vitro fertilization outcome in PCOS patients undergoing controlled ovarian hyperstimulation with different GnRH-analogue? Gynecol Endocrinol 28: 422-424, 2012.

7. Rickes D, Nickel I, Kropf S and Kleinstein J: Increased pregnancy rates after ultralong postoperative therapy with gonadotropin-releasing hormone analogs in patients with endometriosis. Fertil Steril 78: 757-762, 2002.

8. Surrey ES, Silverberg KM, Surrey MW and Schoolcraft WB: Effect of prolonged gonadotropin-releasing hormone agonis therapy on the outcome of in vitro fertilization-embryo transfer in patients with endometriosis. Fertil Steril 78: 699-704, 2002.

9. Mijatovic V, Florijn E, Halim N, Schats R and Hompes P: Adenomyosis has no adverse effects on IVF/ICSI outcomes in women with endometriosis treated with long-term pituitary down-regulation before IVF/ICSI. Eur J Obstet Gynecol Reprod Biol 151: 62-65, 2010.

10. Rotterdam ESHRE/ASRM-Sponsored PCOS Consensus Workshop Group: Revised 2003 consensus on diagnostic criteria and long-term health risks related to polycystic ovary syndrome (PCOS). Hum Reprod 19: 41-47, 2004.

11. Alper MM, Brinsden PR, Fischer R and Wikland M: Is your IVF programme good? Hum Reprod 17: 8-10, 2002.

12. Gonen $\mathrm{Y}$ and Casper RF: Prediction of implantation by the sonographic appearance of the endometrium during controlled ovarian stimulation for in vitro fertilization (IVF). J In Vitro Fert Embryo Transf 7: 146-152, 1990.

13. Chien LW, Au HK, Chen PL, Xiao J and Tzeng CR: Assessment of uterine receptivity by the endometrial-subendometrial blood flow distribution pattern in women undergoing in vitro fertilization-embryo transfer. Fertil Steril 78: 245-251, 2002.

14. Bosch E, Valencia I, Escudero E, Crespo J, Simón C, Remohí J and Pellicer A: Premature luteinization during gonadotropin-releasing hormone antagonist cycles and its relationship with in vitro fertilization outcome. Fertil Steril 80: 1444-1449, 2003

15. Elgindy EA: Progesterone level and progesterone/estradiol ratio on the day of hCG administration: Detrimental cutoff levels and new treatment strategy. Fertil Steril 95: 1639-1644, 2011.

16. Wu Z, Li R, Ma Y, Deng B, Zhang X, Meng Y, Chen X, Liu P and Qiao J: Effect of HCG-day serum progesterone and oestradiol concentrations on pregnancy outcomes in GnRH agonist cycles. Reprod Biomed Online 24: 511-520, 2012.

17. Papanikolaou EG, Pados G, Grimbizis G, Bili E, Kyriazi L, Polyzos NP, Humaidan P, Tournaye $\mathrm{H}$ and Tarlatzis $\mathrm{B}$ : GnRH-agonist versus GnRH-antagonist IVF cycles: Is the reproductive outcome affected by the incidence of proges terone elevation on the day of HCG triggering? A randomized prospective study. Hum Reprod 27: 1822-1828, 2012.

18. Venetis CA, Kolibianakis EM, Bosdou JK and Tarlatzis BC Progesterone elevation and probability of pregnancy after IVF: A systematic review and meta-analysis of over 60000 cycles. Hum Reprod Update 19: 433-457, 2013.
19. Humaidan P, Bungum L, Bungum M and Andersen CY: Ovarian response and pregnancy outcome related to mid-follicular $\mathrm{LH}$ levels in women undergoing assisted reproduction with $\mathrm{GnRH}$ agonist down-regulation and recombinant FSH stimulation. Hum Reprod 17: 2016-2021, 2002.

20. Sharara FI, Lim J and McClamrock HD: Endometrial pattern on the day of oocyte retrieval is more predictive of implantation success than the pattern or thickness on the day of hCG administration. J Assist Reprod Genet 16: 523-528, 1999.

21. Zhang X, Chen CH, Confino E, Barnes R, Milad M and Kazer RR: Increased endometrial thickness is associated with improved treatment outcome for selected patients undergoing in vitro fertilization-embryo transfer. Fertil Steril 83: 336-340, 2005.

22. Järvelä IY, Sladkevicius $P$, Kelly $S$, Ojha K, Campbell S and Nargund G: Evaluation of endometrial receptivity during in-vitro fertilization using three-dimensional power Doppler ultrasound. Ultrasound Obstet Gynecol 26: 765-769, 2005.

23. Kinay T, Tasci Y, Dilbaz S, Cinar O, Demir B and Haberal A: The relationship between endometrial thickness and pregnancy rates in GnRH antagonist down-regulated ICSI cycles. Gynecol Endocrinol 26: 833-837, 2010.

24. Okohue JE, Onuh SO, Ebeigbe P, Shaibu I, Wada I, Ikimalo JI and Okpere EE: The effect of endometrial thickness on in vitro fertilization (IVF)-embryo transfer/intracytoplasmic sperm injection (ICSI) outcome. Afr J Reprod Health 13: 113-121, 2009.

25. Rashidi BH, Sadeghi M, Jafarabadi M and Tehrani Nejad ES: Relationships between pregnancy rates following in vitro fertilization or intracytoplasmic sperm injection and endometrial thickness and pattern. Eur J Obstet Gynecol Reprod Biol 120: 179-184, 2005.

26. Traub ML, Van Arsdale A, Pal L, Jindal S and Santoro N: Endometrial thickness, Caucasian ethnicity, and age predict clinical pregnancy following fresh blastocyst embryo transfer: A retrospective cohort. Reprod Biol Endocrinol 7: 33, 2009.

27. Lintsen AM, Pasker-de Jong PC, de Boer EJ, Burger CW, Jansen CA, Braat DD and van Leeuwen FE: Effects of subfertility cause, smoking and body weight on the success rate of IVF. Hum Reprod 20: 1867-1875, 2005.

28. Raine-Fenning NJ, Campbell BK, Kendall NR, Clewes JS and Johnson IR: Quantifying the changes in endometrial vascularity throughout the normal menstrual cycle with three-dimensional power Doppler angiography. Hum Reprod 19: 330-338, 2004

29. Jungheim ES, Lanzendorf SE, Odem RR, Moley KH, Chang AS and Ratts VS: Morbid obesity is associated with lower clinical pregnancy rates after in vitro fertilization in women with polycystic ovary syndrome. Fertil Steril 92: 256-261, 2009.

30. Metwally M, Cutting R, Tipton A, Skull J, Ledger WL and Li TC: Effect of increased body mass index on oocyte and embryo quality in IVF patients. Reprod Biomed Online 15: 532-538, 2007.

31. Sneed ML, Uhler ML, Grotjan HE, Rapisarda JJ, Lederer KJ and Beltsos AN: Body mass index: Impact on IVF success appears age-related. Hum Reprod 23: 1835-1839, 2008. 